\title{
Development of a performance model for virtual reality tumor resections
}

\author{
Robin Sawaya, MSc, ${ }^{1}$ Ghusn Alsideiri, MD, MSc, ${ }^{1,2}$ Abdulgadir Bugdadi, MD, MSc, ${ }^{1,3}$ \\ Alexander Winkler-Schwartz, MD, ${ }^{1}$ Hamed Azarnoush, PhD, ${ }^{4}$ \\ Khalid Bajunaid, MD, MSc, MMgmt, FRCSC, ${ }^{1,5}$ Abdulrahman J. Sabbagh, MBCHB, FRCSC, ${ }^{1,6,7}$ and \\ Rolando Del Maestro, MD, PhD ${ }^{1}$
}

\begin{abstract}
${ }^{1}$ Neurosurgical Simulation Research and Training Centre, Department of Neurology \& Neurosurgery, McGill University, Montreal, Quebec, Canada; 'Department of Surgery, College of Medicine, Sultan Qaboos University, Muscat, Oman; ${ }^{3}$ Department of Surgery, Faculty of Medicine, Umm Al-Qura University, Mecca, Saudi Arabia; ${ }^{4}$ Department of Biomedical Engineering, Amirkabir University of Technology (Tehran Polytechnic), Tehran, Iran; ${ }^{5}$ Divison of Neurosurgery, Faculty of Medicine, University of Jeddah, Saudi Arabia; ${ }^{6}$ Department of Neurosurgery, National Neuroscience Institute, King Fahad Medical City, Riyadh, Saudi Arabia; and 'Division of Neurosurgery, Department of Surgery, Faculty of Medicine and Clinical Skill and Simulation Center, King Abdulaziz University, Jeddah, Saudi Arabia
\end{abstract}

OBJECTIVE Previous work from the authors has shown that hand ergonomics plays an important role in surgical psychomotor performance during virtual reality brain tumor resections. In the current study they propose a hypothetical model that integrates the human and task factors at play during simulated brain tumor resections to better understand the hand ergonomics needed for optimal safety and efficiency. They hypothesize that 1) experts (neurosurgeons), compared to novices (residents and medical students), spend a greater proportion of their time in direct contact with critical tumor areas; 2) hand ergonomic conditions (most favorable to unfavorable) prompt participants to adapt in order to optimize tumor resection; and 3) hand ergonomic adaptation is acquired with increasing expertise.

METHODS In an earlier study, experts (neurosurgeons) and novices (residents and medical students) were instructed to resect simulated brain tumors on the NeuroVR (formerly NeuroTouch) virtual reality neurosurgical simulation platform. For the present study, the simulated tumors were divided into four quadrants (Q1 to Q4) to assess hand ergonomics at various levels of difficulty. The spatial distribution of time expended, force applied, and tumor volume removed was analyzed for each participant group (total of 22 participants).

RESULTS Neurosurgeons spent a significantly greater percentage of their time in direct contact with critical tumor areas. Under the favorable hand ergonomic conditions of Q1 and Q3, neurosurgeons and senior residents spent significantly more time in Q1 than in Q3. Although forces applied in these quadrants were similar, neurosurgeons, having spent more time in Q1, removed significantly more tumor in Q1 than in Q3. In a comparison of the most favorable (Q2) to unfavorable (Q4) hand ergonomic conditions, neurosurgeons adapted the forces applied in each quadrant to resect similar tumor volumes. Differences between Q2 and Q4 were emphasized in measures of force applied per second, tumor volume removed per second, and tumor volume removed per unit of force applied. In contrast, the hand ergonomics of medical students did not vary across quadrants, indicating the existence of an "adaptive capacity" in neurosurgeons.

CONCLUSIONS The study results confirm the experts' (neurosurgeons) greater capacity to adapt their hand ergonomics during simulated neurosurgical tasks. The proposed hypothetical model integrates the study findings with various human and task factors that highlight the importance of learning in the acquisition of hand ergonomic adaptation.

https://thejns.org/doi/abs/10.3171/2018.2.JNS172327

KEYWORDS ergonomics; neurosurgery; NeuroTouch/NeuroVR; simulation; virtual reality; tumor resection; performance metrics; surgical technique

ABBREVIATIONS PGY = postgraduate year; $Q 1, \ldots, Q 4=$ quadrant $1, \ldots$, quadrant 4.

SUBMITTED September 16, 2017. ACCEPTED February 16, 2018.

INCLUDE WHEN CITING Published online August 3, 2018; DOI: 10.3171/2018.2.JNS172327. 
$\mathrm{T}$ HE NeuroVR (formerly NeuroTouch, CAE Healthcare) virtual reality neurosurgical simulation platform has allowed us to deconstruct and evaluate the surgical performance of "expert" and "novice" participants carrying out virtual reality brain tumor resections. ${ }^{2-7,11,12,14,21,23}$ The performance of neurosurgeon, resident, and medical student groups has been studied using metrics developed by our group. These metrics focus on psychomotor skills such as safety, quality, efficiency, and bimanual dexterity. ${ }^{2-7,23}$ The recent development of the force pyramid, a visual representation of the spatial distribution of forces applied during virtual reality brain tumor resections, has allowed us to assess hand ergonomics in groups with different levels of expertise. These results have allowed us to identify critical areas of the tumor that require participants to adapt to task constraints in order to complete the resection procedure safely and efficiently. ${ }^{6,21}$

The ability to adapt hand ergonomics, the position and orientation of the hand, wrist, and fingers, to the physical constraints of the tumor plays an important role in surgical performance. Hand ergonomics has been studied in the context of laparoscopic surgeries, where a link has been established between hand and wrist positions and overall surgical performance..$^{8-10,16}$ In the present study, we use spatial distribution analysis to investigate the effects of hand ergonomics on metrics such as total time expended, total force applied, total tumor volume removed, mean force applied per second, mean tumor volume removed per second, and mean tumor volume removed per unit of force applied.

Given the results from this study, along with our previous findings and relevant information from the literature, we propose a hypothetical model for surgical performance in simulated brain tumor resections. This model integrates human factors, such as inherent ability and acquired experience, and task factors, such as visual and haptic information from tumor characteristics and stress incurred, to describe the effects of operator hand ergonomics on safety and efficiency. This framework highlights the importance of adaptation in hand ergonomics and identifies several factors that are hypothesized to influence surgical performance in virtual reality brain tumor resections.

Several predictions were tested in order to develop the proposed hypothetical model of virtual reality surgical performance: 1) experts (neurosurgeons), as compared to novices (residents and medical students), spend a greater proportion of their time in direct contact with critical tumor areas; 2) hand ergonomic conditions (from most favorable to unfavorable) prompt participants to adapt in order to optimize tumor resection; and 3) hand ergonomic adaptation is acquired with increasing expertise.

\section{Methods}

\section{Subjects}

Six neurosurgeons, 6 senior residents (PGY4-6), 6 junior residents (PGY1-3), and 6 medical students participated in an earlier study. To control for the variable of experience in neurosurgical simulation, only participants who had been enrolled in a previous NeuroVR study using similar scenarios were included in that study. Since significant differences were previously observed between right- and left-handed participants, we removed left-handed individuals (one senior resident and one medical student) from subsequent analyses, leaving data for 6 neurosurgeons, 5 senior residents, 6 junior residents, and 5 medical students (22 total participants) for the present study. ${ }^{6,21}$ Everyone had signed a consent form approved by McGill's ethics review board prior to the trial.

\section{NeuroVR}

The NeuroVR virtual reality neurosurgical simulation platform was used in earlier studies. ${ }^{27,11,12,14,21,23}$ Simulated tumor resections were performed with an aspirator in the dominant hand and a sucker in the nondominant hand to control bleeding (Fig. 1A). Instrument intensities were controlled and set at constant values. Participants were instructed verbally and in writing to resect a series of identical tumors ( 2 minutes were allowed per tumor) with minimal injury to surrounding normal tissue (see Video 1 in Bajunaid et al. ${ }^{7}$ ).

\section{Simulation Scenarios}

Data in the present study were collected in an earlier study on surgical performance. ${ }^{7}$ Data for the initial 3 tumors (out of a series of 6) in that study were used here. The tumors were identical in shape (ellipsoids), color (gliomalike), and stiffness (Young's modulus $9 \mathrm{kPa}$ ) and were homogeneous throughout. All had multiple bleeding points and were embedded in a white matter-like background (Young's modulus $3 \mathrm{kPa}$; Fig. 1B).

The surgical field was separated into three areas (Fig. 1C): nontumor components (area A), tumor above the brain surface (area B), and tumor below the brain surface (area C). To assess spatial distribution, the tumor (top view) was further partitioned into 4 equal quadrants (Q1 to Q4, counter-clockwise from the top right quadrant; Fig. 1D). Based on our previous results, three hand ergonomic conditions were identified: minimal wrist flexion in Q2, slightly increased wrist flexion in Q1 and Q3, and maximal wrist flexion and internal rotation in $\mathrm{Q} 4{ }^{6,21}$

\section{Data Analyses}

Data used in this project included the position of the dominant-hand instrument in space (xyz position coordinates), as well as the time in seconds (s), force applied in Newtons $(\mathrm{N})$, and tumor tissue removed in cubic centimeters (cc) corresponding to each position. For each participant, the first resection was considered as a practice trial and was therefore discarded. All analyses were performed using MATLAB (MathWorks).

An initial analysis determined the proportion of the total time that participants held the tip of the aspirator in critical surgical field area $\mathrm{C}$ (compared to surgical field areas $\mathrm{A}+\mathrm{B}$ ).

For each tumor resection completed by a participant, the total time expended (s), total force applied (N), and total tumor volume removed (cc) per quadrant (Q1-Q4) were calculated. Each participant's values for the metrics were averaged over the two tumors analyzed.

Three additional metrics were derived from the values above: mean force applied per second (N/s), mean tumor 


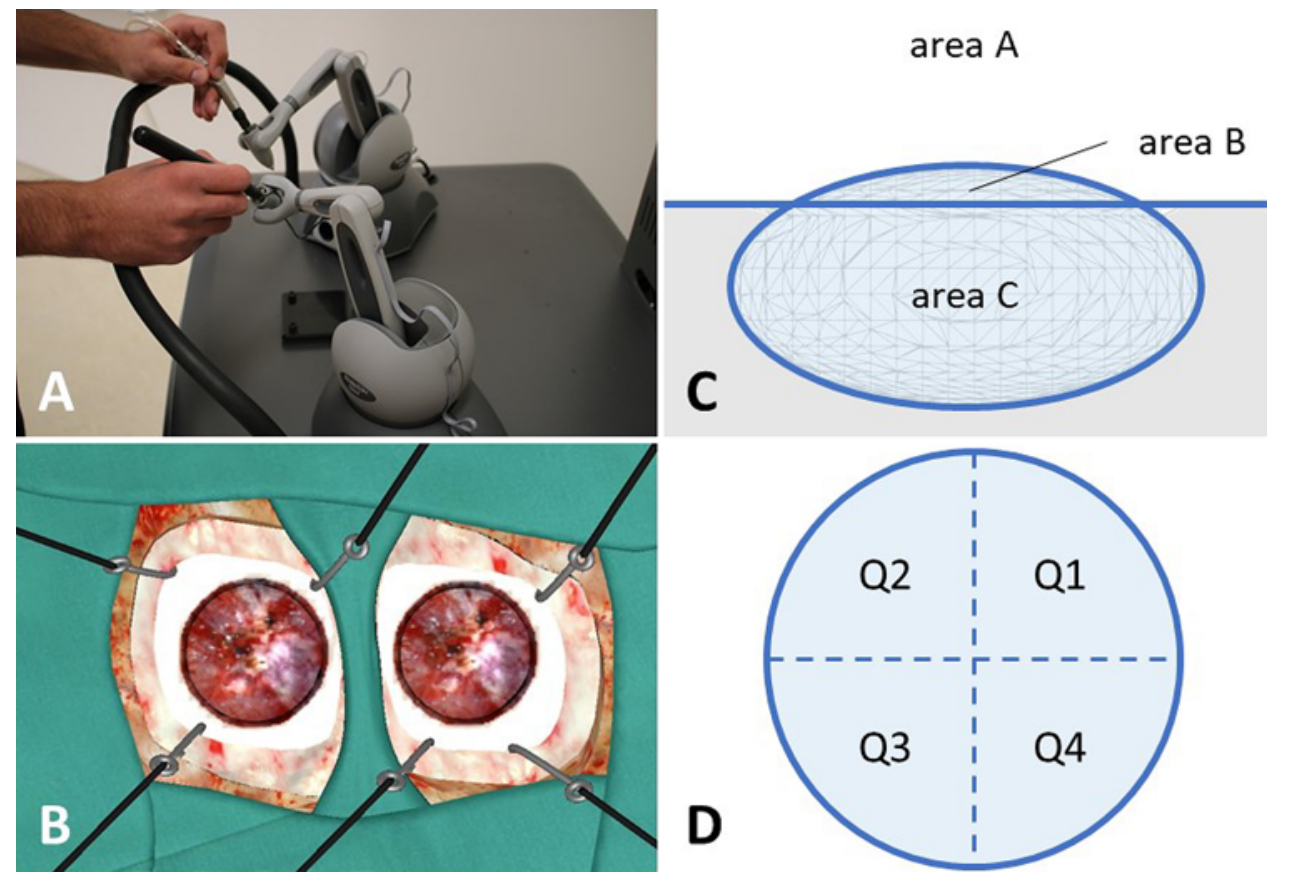

FIG. 1. Study design. A: Instrument setup. To carry out the resection, participants held a simulated aspirator in their dominant hand (right), and to control the bleeding, they held a sucker in their nondominant hand (left). B: The simulated tumors (top view) presented to the participants were identical in color (glioma-like) and stiffness (Young's modulus $9 \mathrm{kPa}$ ) and were embedded in a white matter-like background (Young's modulus $3 \mathrm{kPa}$ ). C: Surgical field areas (side view). Cross-section of a tumor presented to participants. Three surgical field areas can be identified: area A corresponds to all components that are not the tumor; area B corresponds to tumor above the surface of the brain; area C corresponds to tumor below the surface of the brain. D: Tumors are divided into four quadrants (top view) presented in a counter-clockwise fashion. These divisions extend through the depth of the tumor. Figure is available in color online only.

volume removed per second (cc/s), and mean tumor volume removed per unit of force applied $(\mathrm{cc} / \mathrm{N})$. Values for mean force applied and mean tumor volume removed for each group were represented as scatter plots with the associated $95 \%$ confidence ellipse.

\section{Statistical Analyses}

Statistical analyses were conducted using JMP statistical software (SAS Institute). A nonparametric approach using the Kruskal-Wallis test, followed by Dunn's test for pairwise comparisons, was chosen due to the small sample sizes. A p value below 0.05 was considered significant. Error bars show the standard error.

\section{Results \\ Demographics}

The mean age ( \pm standard error) was $41.7 \pm 3.0$ years for neurosurgeons, $31.5 \pm 1.0$ for senior residents, $28.5 \pm$ 0.8 for junior residents, and $23.4 \pm 0.4$ for medical students. Neurosurgeons had $7.3 \pm 1.9$ years of surgical practice experience. All participants were right handed and $86 \%$ were male.

\section{Time Expended per Tumor Area}

Because of its importance in the safety of the procedure, the proportion of time spent by participant groups in surgical field area $\mathrm{C}$ was compared to that in $\mathrm{A}$ and B combined (Fig. 2). Neurosurgeons spent a significantly greater proportion of their time in surgical field area $\mathrm{C}$ (tumor below the brain surface). A similar trend can be observed for the senior resident group. However, the proportion of time spent within surgical field area $C$ was not significantly different between all groups. Because of its critical location adjacent to surrounding normal tissue and

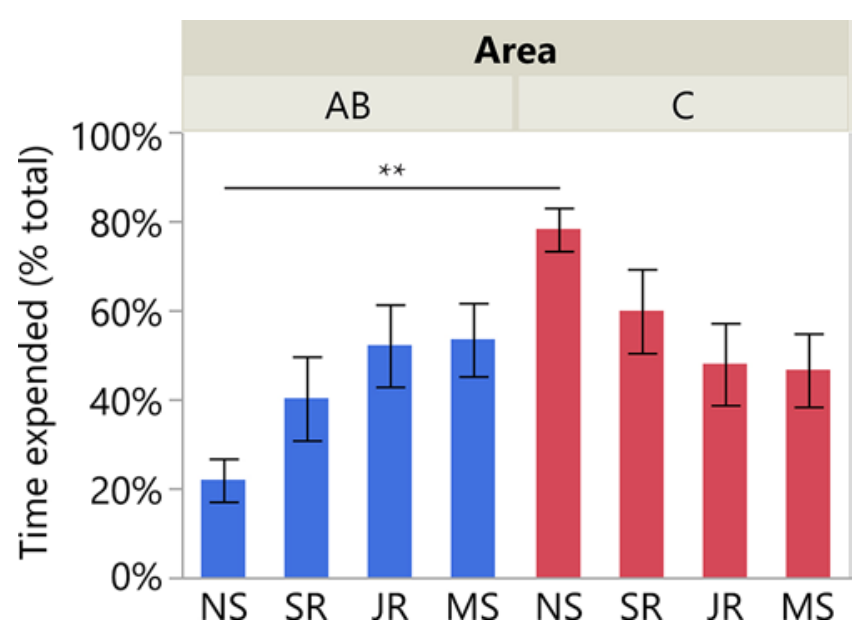

FIG. 2. Time expended per area. The percent of the total time spent in each area is shown for all participant groups: neurosurgeons (NS), $n$ $=6$; senior residents (SR), $n=5$; junior residents (JR), $n=6$; medical students (MS), $n=5 .{ }^{*} p<0.05$ or ${ }^{* *} p<0.01$. Figure is available in color online only. 


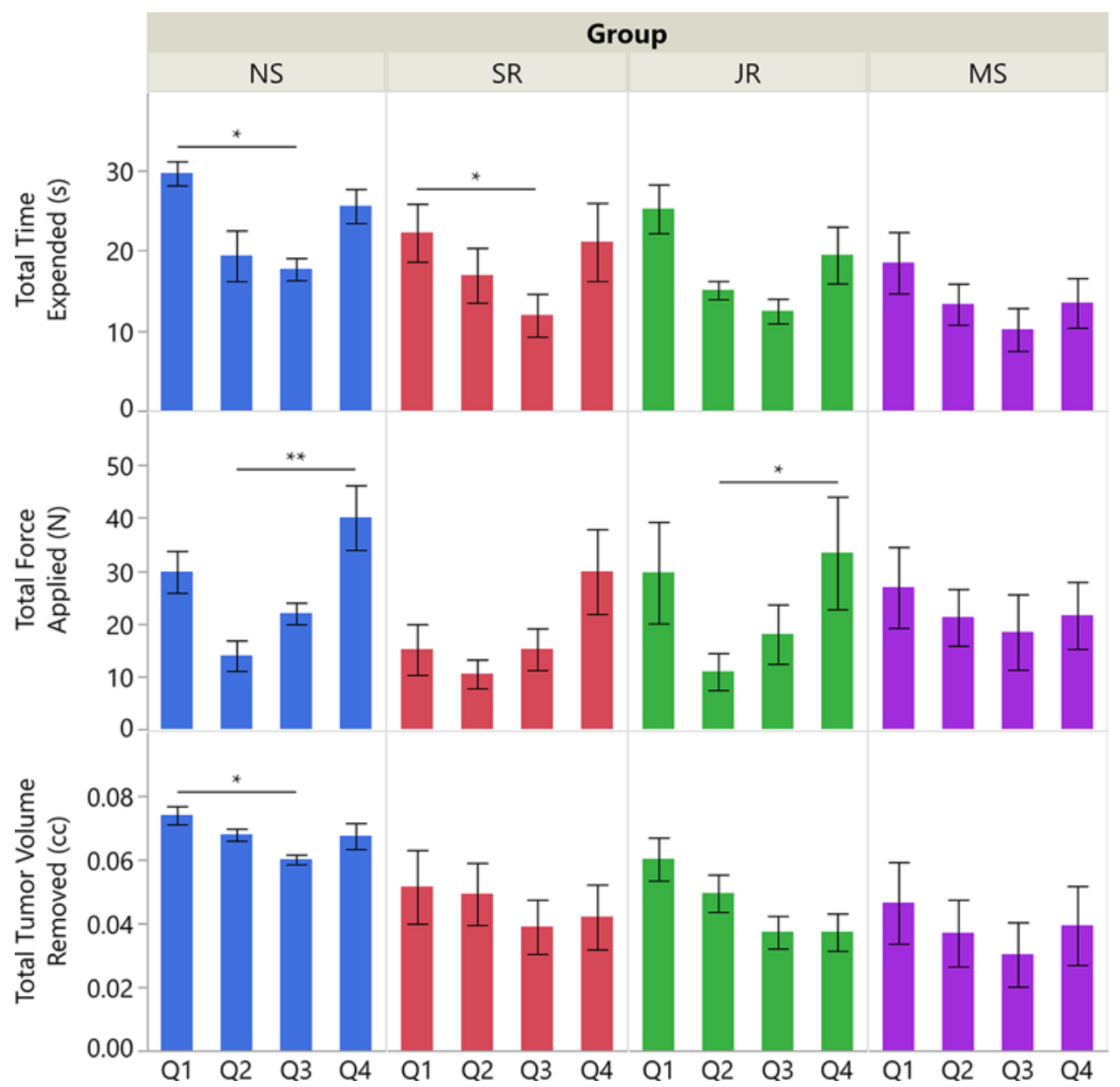

FIG. 3. Simple metrics. The total time expended (s), total force applied (N), and total volume of tumor removed (cc) are calculated per quadrant for each group: neurosurgeons (NS), $n=6$; senior residents (SR), $n=5$; junior residents (JR), $n=6$; medical students (MS), $n=5 .{ }^{*} p<0.05$ or ${ }^{* *} p<0.01$. Figure is available in color online only.

its importance in the safety of the procedure, only area $\mathrm{C}$ was considered in the following analyses.

\section{Quadrants}

Three initial simple metrics were assessed: total time expended (s), total force applied (N), and total volume removed (cc). Total time expended was significantly higher in Q1 than in Q3 for neurosurgeons and senior residents (Fig. 3). Although the relationship was nonsignificant, neurosurgeons and resident groups appeared to spend less time in the left quadrants (Q2 and Q3) than in the right quadrants (Q1 and Q4). Total force applied was significantly higher in Q4 (unfavorable hand ergonomics) than in Q2 (most favorable hand ergonomics) for neurosurgeons, as seen in our previous studies. ${ }^{6,21}$ Resident groups followed a similar trend. Total tumor volume removed was significantly greater in Q1 than in Q3 for neurosurgeons.

To factor out the amount of time spent per quadrant, the total force applied and total volume removed were divided by the total time expended. These advanced metrics, along with the mean tumor volume removed per unit of force applied, allowed us to observe the participants' average hand ergonomics in each quadrant. Mean force applied per second (N/s) was significantly higher in Q4 than in Q2 for neurosurgeons, with a similar trend observed in the resident groups (Fig. 4). The mean tumor volume removed per second (cc/s) was significantly greater in Q2 than in Q4 for junior residents, with a similar trend observed for neurosurgeons and senior residents. Finally, the mean tumor volume removed per unit of force applied $(\mathrm{cc} / \mathrm{N})$ was significantly greater in Q2 than in Q4 for neurosurgeons and junior residents, with a similar trend observed for senior residents.

Medical students' values for all metrics remained constant across all four quadrants (Figs. 3 and 4), suggesting a lack of adaptive behavior when faced with different physical and situational constraints during the simulated neurosurgical procedures.

\section{Ellipses}

Since the most significant differences were observed between Q2 (most favorable hand ergonomic conditions) and Q4 (unfavorable hand ergonomic conditions) for force applied, mean force applied per second, and mean tumor volume removed per unit of force applied (with a similar trend observed in mean tumor volume per second), these quadrants were selected for our qualitative analysis of each group's "adaptive capacity." The mean force applied per 


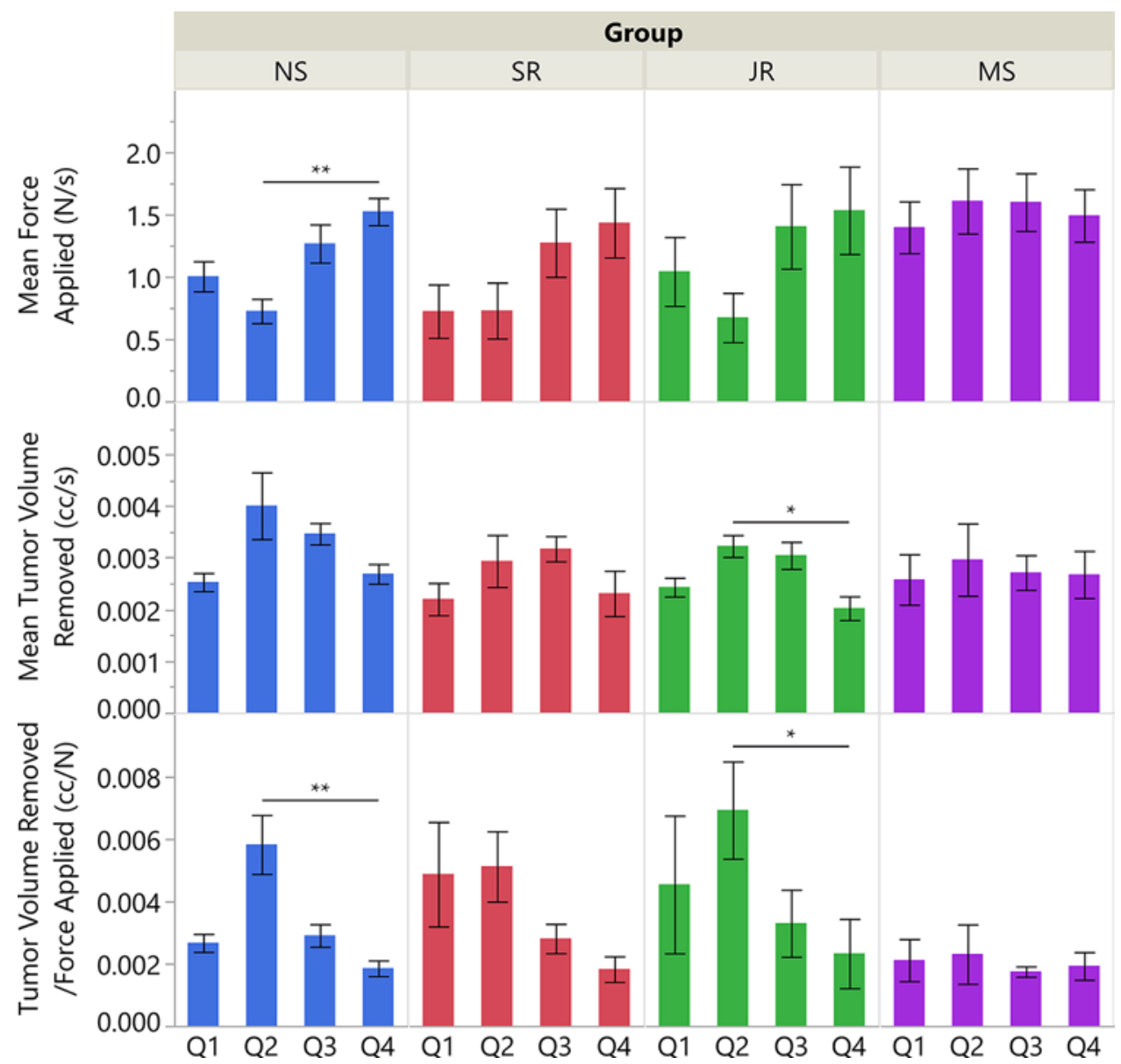

FIG. 4. Advanced metrics. The mean force applied per second (N/s), mean volume of tumor removed per second (cc/s), and mean tumor volume removed per unit of force applied (cc/N) are calculated per quadrant for each group: neurosurgeons (NS), $n=6$; senior residents (SR), $n=5$; junior residents $(J R), n=6$; medical students $(M S), n=5$. ${ }^{*} p<0.05$ or ** $p<0.01$. Figure is available in color online only.

second was plotted against the mean tumor volume removed per second for each group, and the $95 \%$ confidence ellipse for each quadrant was outlined (Fig. 5). The ellipses for Q2 and Q4 segregated into two distinct groups for neurosurgeons, overlapped to a small extent for residents, and overlapped completely for medical students.

\section{Groups}

Significant differences were observed for the tumor volume removed between neurosurgeons and junior residents in Q2 and Q3, and between neurosurgeons and medical students in Q2 and Q3.

\section{Discussion}

\section{Summary}

Our group has developed and assessed several metrics to define expert surgical psychomotor performance during virtual reality tumor resections. ${ }^{2-7,11,21,23}$ Given results of these studies, the relevant literature, and the present findings, we propose a hypothetical model that integrates human and task factors to understand hand ergonomic variations and their subsequent effect on operator safety and efficiency.
Data in the present study demonstrate that neurosurgeons have developed a greater capacity to adapt their hand ergonomics to perform tumor resections safely and efficiently. The evolution of the spatial distributions of time, force, and volume with increasing expertise indicates the behavioral learning of residents following their residency training. This capacity to adapt hand ergonomics plays an important role in expert surgical psychomotor performance.

\section{Quadrants}

In previous studies, our group had shown significant differences between Q2 and Q4 in terms of the amount of force applied, with all participant groups applying more force in Q4 with their dominant hand. ${ }^{6,21}$ These results were hypothesized to be attributable to the specific hand ergonomics needed to resect tumor in each quadrant. With the aspirator in the dominant hand, the participant's wrist is in a favorable neutral position when resecting in Q2 and in an unfavorable flexed position while in Q4. Therefore, each of the four quadrants requires different hand ergonomics to carry out the tumor resection. According to our current observations and those made in previous studies, the quad- 


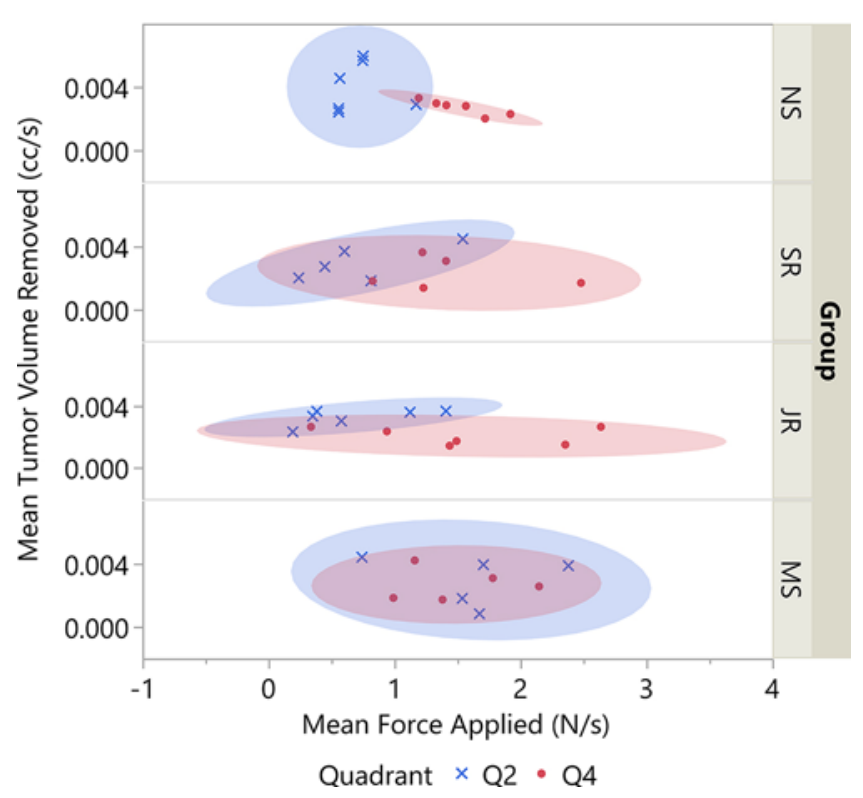

FIG. 5. The 95\% confidence ellipses show the spread and relationship between the mean force applied per second $(\mathrm{N} / \mathrm{s})$ and the mean volume of tumor removed per second (cc/s) for Q2 and Q4, the quadrants that are most significantly different for each group: neurosurgeons (NS), $n=$ 6; senior residents (SR), $n=5$; junior residents (JR), $n=6$; medical students (MS), $n=5$. There is minimal overlap between the two quadrants for neurosurgeons in comparison to those for the other groups. Figure is available in color online only.

rants create three hand ergonomic conditions: most favorable (Q2), favorable (Q1 and Q3), and unfavorable (Q4). ${ }^{6,21}$

To address the hypothesis that the various quadrants induce different degrees of hand ergonomic adaptation in participants, we assessed simple metrics: time expenditure, force application, and tumor volume removal in each quadrant (Fig. 3). Under favorable hand ergonomic conditions $(\mathrm{Q} 1$ and $\mathrm{Q} 3)$, the tumor volume removed is more closely related to the time expended: neurosurgeons applied similar forces in the two quadrants but spent more time in Q1 than in Q3 and therefore removed a greater volume of tumor in Q1.

To address the prediction that hand ergonomic conditions prompt participants to adapt in order to optimize tumor resection, we compared the results under the most favorable (Q2) and unfavorable (Q4) hand ergonomic conditions. We observed that neurosurgeons significantly increased their force application in Q4, corroborating our previous findings. ${ }^{6,21}$ Since time expenditure and tumor volume removal were not significantly different between these quadrants, we can infer the existence of an adaptive behavior in force application that allows neurosurgeons to achieve the same result despite variable conditions.

There are a few possible explanations for this outcome. First, the increase in force application from Q2 to Q4 may be due to neurosurgeons adapting their hand ergonomics to achieve equivalent tumor removal in both quadrants. Second, the difference in force applied between the two quadrants may be the result of the unfavorable hand ergonomic conditions of Q4, which force participants to adopt a flexed wrist position. It has been shown that a flexed wrist can reduce one's ability to perceive forces and therefore lead them to increase force use..$^{13,17,20}$ The neurosurgeons' experience may allow them to appreciate the discrepancy between their sensory feedback and the real stiffness of the tumor, thus prompting them to adjust their force application. Under unfavorable hand ergonomic conditions, this conscious or subconscious adaptation allows neurosurgeons to achieve their desired result.

The metrics of mean force applied per second and mean tumor volume removed per second (Fig. 4) emphasize the differences in hand position and ergonomics between Q2 and Q4. The unfavorable conditions in Q4 lead to an increase in mean force application per second, which indicates decreased safety, and a decrease in mean tumor volume removed per second, which indicates decreased efficiency. Conversely, in Q2, neurosurgeons apply very low mean forces per second and remove a greater mean volume of tumor per second. Therefore, in the most favorable hand ergonomic conditions, experts maximize both the safety and the efficiency of their movements. Adaptation of hand ergonomics is most evident when assessing mean tumor volume removed per unit of force applied. Neurosurgeons are the least efficient in Q4, removing very little volume with each Newton of force applied, yet they remove a volume of tumor equivalent to that in Q2.

\section{Participant Groups}

Comparing neurosurgeon and resident groups to the medical students suggests that greater neurosurgical exposure results in increased adaptation of the former groups to the constraints that each tumor quadrant imposes on their hand ergonomics. Medical student behavior does not change between quadrants, and these results imply that adaptation is a learned skill, as seen in the spatial distribution of the neurosurgeons' metrics. The 95\% confidence ellipses further highlight the differences between experts (neurosurgeons) and novices (residents and medical students) in their capacity to adapt their surgical behavior to the location of the tumor resection. The two discrete ellipses observed for neurosurgeons clearly differentiate the forces applied and the tumor volumes removed when operating in Q2 and Q4. These same ellipses overlap completely for medical students and begin to separate for junior and senior residents, indicating a progression of skill acquisition. Given that the simulator and its constraints were identical for all participants, adaptation of hand ergonomics appears to be an intrinsic ability rather than the result of extrinsic factors.

\section{Hand Ergonomics}

The overall results confirm the significant role of hand ergonomics in the surgical psychomotor performance of the groups assessed. Increasing expertise correlates with a greater capacity for hand ergonomic adaptation. The differences observed between Q2 and Q4 are an indicator of this behavior. Studies in various disciplines have linked hand ergonomics to performance. In pianists, it has been shown that tempo and evenness of a musical performance are directly correlated with wrist mobility.$^{18}$ In laparoscopic surgery, procedures involving excessive wrist flexion resulted in increased fatigue and decreased overall performance. $^{8-10,16}$ 
TABLE 1. Summary of results observed in the expert (neurosurgeon) group

\begin{tabular}{clcccccc}
\hline Quadrant & $\begin{array}{c}\text { Hand Ergonomic } \\
\text { Conditions }\end{array}$ & $\begin{array}{c}\text { Time } \\
\text { Expended (s) }\end{array}$ & $\begin{array}{c}\text { Force } \\
\text { Applied (N) }\end{array}$ & $\begin{array}{c}\text { Mean Force } \\
\text { Applied (N/s) }\end{array}$ & $\begin{array}{c}\text { Mean Tumor Vol } \\
\text { Removed (cc/s) }\end{array}$ & $\begin{array}{c}\text { Mean Tumor Vol Removed/ } \\
\text { Force Applied (cc/N) }\end{array}$ & $\begin{array}{c}\text { Tumor Vol } \\
\text { Removed (cc) }\end{array}$ \\
\hline Q1 & Favorable & High & Low & Low & Low & Low & High \\
\hline Q3 & Favorable & Low & Low & Low & Low & Low & Low \\
\hline Q2 & Most favorable & Low & Low & Low & High & High & Low \\
\hline Q4 & Unfavorable & High & High & High & Low & High \\
\hline
\end{tabular}

Q1 is compared to Q3 (both favorable hand ergonomic conditions) and Q2 is compared to Q4 (most favorable and unfavorable hand ergonomic conditions, respectively). Low values for time expended, force applied, and mean force applied indicate safe brain tumor removal. High values for mean tumor volume removed and mean tumor volume removed per unit of force applied indicate efficient brain tumor removal. The resulting tumor volume removed values are high when the outcome is considered successful and low when the outcome is otherwise. Adaptation of the neurosurgeons' hand ergonomics occurs in Q2 and Q4 such that despite reduced efficiency and safety in Q4, they are able to achieve a similar surgical outcome.

Visser et al. showed that the greater concentration needed to perform exact and calculated movements was correlated with increased muscle contraction in participants performing a computer task with a mouse. ${ }^{22}$ The greater muscle activity allowed users to control unwanted movements, but also led them to apply greater forces with their fingers on the mouse. Therefore, wrist flexion and increased muscle activity, secondary to cognitive stress and a need for precision, lead to decreased wrist mobility, which can adversely impact performance. This may provide an explanation for the location of greatest forces in Q4, at the tumor-normal tissue interface, where precision is crucial to avoid damaging the surrounding normal tissue. ${ }^{21}$ In the same study by Visser and colleagues, it was shown that precision also required participants to reduce the speed of their movements when closer to the target. ${ }^{22}$ These observations suggest that it may be useful to investigate quadrant differences in dominant-hand speed to assess the role of precision on hand ergonomics.

Other studies have observed increased times to complete tasks with reduced wrist mobility. ${ }^{1}$ These effects are further compounded by the fact that wrist positions that deviate from a neutral position may affect both sensory and motor nerve function. ${ }^{13,17,20}$ Our results are consistent with the concept that unfavorable hand ergonomic conditions (such as those in Q4) may increase muscle activity and rigidity, which reduces wrist mobility and compromises sensory feedback and motor control. In the end, these conditions result in longer task times, increased applied forces, decreased instrument movement speeds, and thus decreased efficiency.

\section{Virtual Reality Brain Tumor Resection Model}

Results from this study, summarized in Table 1, along with our group's previous findings and literature on ergonomics, can be integrated into a hypothetical model of surgical performance in virtual reality brain tumor resections. This model links human and task factors to hand ergonomics and to the overall safety and efficiency of the procedure (Fig. 6).

Human factors such as inherent ability and acquired experience (during residency and beyond) determine an individual's surgical expertise, the automaticity with which they perform a procedure, and their overall surgical fingerprint. ${ }^{11,15,21,23}$ When tested on the NeuroVR, applicants to neurosurgery residency split into top, middle, and bottom performance groups, proving the existence of an inherent ability. ${ }^{23}$ Automaticity was similarly assessed on the simulator, showing that neurosurgeons, as compared to residents, were consistent in their approach when tasked with resecting identical tumors. ${ }^{11}$ The study by Bugdadi et al.," ${ }^{11}$ along with our work on force pyramids, led to the concept of "surgical fingerprint," the idea that each individual possesses a unique style in simulated brain tumor resections. ${ }^{6,21}$

Task factors are dynamic, continually changing as the operator progresses through the simulation. In previous investigations, characteristics such as tumor stiffness and color have been shown to affect the amount of force applied by novices and experts..$^{2,6,11,21}$ Similarly, tumor bleeding and the resulting stress to the operator have been linked to decreased surgical performance on the NeuroVR simulator? The current investigation adds tumor location (based on quadrants) as an additional task factor that the operator must account for. Finally, preprocedural data such as laboratory investigations and scans in the patient file must be considered prior to the operation. Therefore, given the incoming visual and haptic information received and the preprocedural data, operators assess the complexity of the task, determine the need for precise movements, and may consciously or subconsciously experience stress.

Human and task factors are then integrated by the operator, resulting in the adaptation of the hand ergonomics necessary to carry out the simulated procedure. As previously mentioned, operators in the unfavorable quadrant Q4 must maximally flex their wrist to remove the tumor but can maintain a neutral and relaxed wrist when working in the most favorable quadrant Q2. The muscle activation and contraction necessary for wrist flexion influences both sensory and motor functions as well as wrist mobility. ${ }^{13,17,20}$ As seen in the current study, the result of hand ergonomic adaptation affects task time and forces applied. Instrument speed is also hypothesized to play a role in the overall safety and efficiency of the procedure. ${ }^{22}$

This proposed model increases our understanding of the mechanisms behind expert surgical performance under favorable and unfavorable conditions and identifies several hypothetical factors whose exact roles remain to be elucidated. Direct measures of instrument orientation (angle), forearm muscle electromyography, or the use of a glove that determines hand and finger position ${ }^{19}$ are approaches 
HUMAN FACTORS

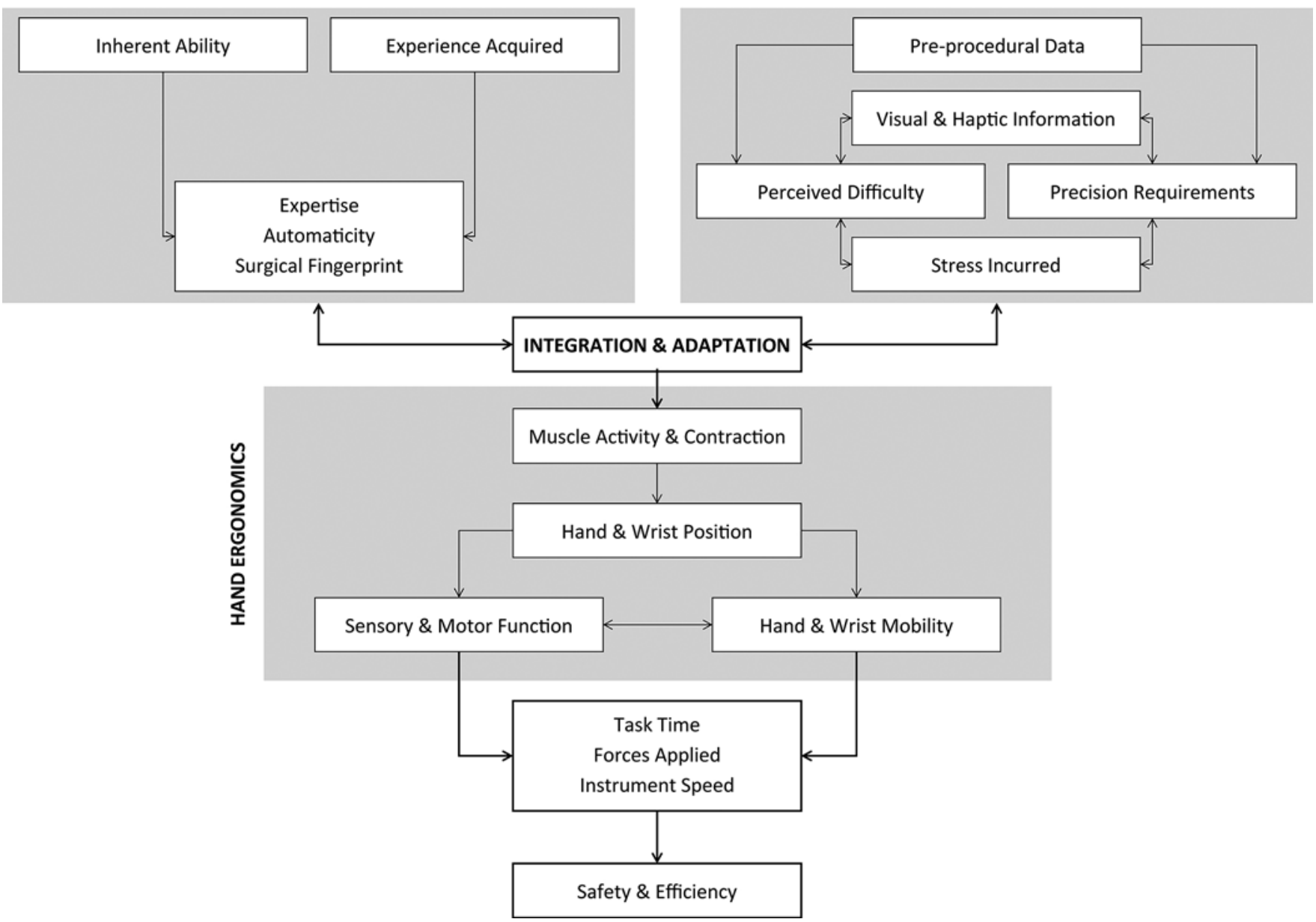

FIG. 6. Hypothetical model of surgical performance in virtual reality brain tumor resections. Diagram representing the effect of various human and task factors, which, after integration and adaptation, produce specific hand ergonomics and result in a surgical performance focused on the overall safety and efficiency of the procedure.

being explored to further test and refine the model. Continued assessment and development are necessary before the model can be incorporated into a curriculum to enhance neurosurgical training.

\section{Limitations}

The NeuroVR virtual reality neurosurgical platform does not represent the complex reality of situations encountered in the operating room. First, in order to simplify the interpretation of participants' surgical performance, we controlled for multiple variables: specific instruments were used, instrument intensities were held constant, microscope movement was not allowed, and participants were not able to change their operative view by moving around the simulated operative field. In an operating room setting, experts can change the instruments used, alter instrument intensities, change their body's position and that of the microscope, and even switch hands when operating under difficult conditions. Our current model does not take into account the range of adjustments available to a neurosurgeon, which may help to decrease the undesirable impact of hand ergonomics. Modifications of the model will be needed to account for these secondary adaptive responses.
Second, because of their significant differences with right-handed participants, left-handed participants were excluded from our study. ${ }^{21}$ However, this prevented us from commenting on their hand ergonomics. Third, the 2-minute time allotment, although sufficient for most participants, represents only a small portion of the time involved in a real tumor resection.

Finally, as a result of the requirement for previous experience on the NeuroVR, the small sample size and similar educational background of all participants make it difficult to confidently extend our results to other study populations.

These concerns are being addressed in work that has gathered a larger sample size, with participants from multiple institutes that were asked to resect an irregularly shaped, and therefore realistic, tumor.

\section{Conclusions}

Hand ergonomics plays an important role in surgical psychomotor performance. Explicit measures of ergonomics are needed to better understand its effects on various metrics, both on the neurosurgical simulation platform and in the operating room. The model established in this study 
may aid future trainees to gain awareness of their movements at an earlier stage and enhance their dexterity to carry out procedures safely and efficiently.

\section{Acknowledgments}

This work was supported by the Di Giovanni Foundation, the Montreal English School Board, the Colannini Foundation, and the Montreal Neurological Institute and Hospital. Robin Sawaya holds the Christian Geada Brain Tumour Research Studentship from the Montreal Neurological Institute. Dr. Rolando Del Maestro is the William Feindel Emeritus Professor in Neuro-Oncology at McGill University.

We thank all the medical students, residents, and neurosurgeons from the Montreal Neurological Institute and Hospital who participated in this study. We also thank Robert DiRaddo, Group Leader, Simulation, Life Sciences Division, National Research Council of Canada at Boucherville, and his team, including Denis Laroche, Valérie Pazos, Nusrat Choudhury, and Linda Pecora, for their support in the development of the scenarios used in these studies and all the members of the Simulation, Life Sciences Division, National Research Council of Canada. Finally, we thank Dr. Roy Dudley for his theoretical input and Mina Khan, Dana Almedallah, and Saad Alghamdi for their support.

\section{References}

1. Adams BD, Grosland NM, Murphy DM, McCullough M: Impact of impaired wrist motion on hand and upper-extremity performance. J Hand Surg Am 28:898-903, 2003

2. Alotaibi FE, AlZhrani GA, Mullah MA, Sabbagh AJ, Azarnoush $\mathrm{H}$, Winkler-Schwartz A, et al: Assessing bimanual performance in brain tumor resection with NeuroTouch, a virtual reality simulator. Neurosurgery 11 (Suppl 2):89-98, 2015

3. Alotaibi FE, AlZhrani GA, Sabbagh AJ, Azarnoush H, Winkler-Schwartz A, Del Maestro RF: Neurosurgical assessment of metrics including judgment and dexterity using the virtual reality simulator NeuroTouch (NAJD metrics). Surg Innov 22:636-642, 2015

4. AlZhrani G, Alotaibi F, Azarnoush H, Winkler-Schwartz A, Sabbagh A, Bajunaid K, et al: Proficiency performance benchmarks for removal of simulated brain tumors using a virtual reality simulator NeuroTouch. J Surg Educ 72:685696, 2015

5. Azarnoush H, Alzhrani G, Winkler-Schwartz A, Alotaibi F, Gelinas-Phaneuf N, Pazos V, et al: Neurosurgical virtual reality simulation metrics to assess psychomotor skills during brain tumor resection. Int J CARS 10:603-618, 2015

6. Azarnoush H, Siar S, Sawaya R, Zhrani GA, WinklerSchwartz A, Alotaibi FE, et al: The force pyramid: a spatial analysis of force application during virtual reality brain tumor resection. J Neurosurg 127:171-181, 2017

7. Bajunaid K, Mullah MA, Winkler-Schwartz A, Alotaibi FE, Fares J, Baggiani M, et al: Impact of acute stress on psychomotor bimanual performance during a simulated tumor resection task. J Neurosurg 126:71-80, 2017

8. Berguer R: The application of ergonomics in the work environment of general surgeons. Rev Environ Health 12:99106, 1997

9. Berguer R: Surgical technology and the ergonomics of laparoscopic instruments. Surg Endosc 12:458-462, 1998

10. Berguer R, Forkey DL, Smith WD: Ergonomic problems associated with laparoscopic surgery. Surg Endosc 13:466468, 1999

11. Bugdadi A, Sawaya R, Olwi D, Al-Zhrani G, Azarnoush H, Sabbagh A, et al: Automaticity of force application during simulated brain tumor resection: testing the Fitts and Posner model. J Surg Educ 75:104-115, 2018
12. Delorme S, Laroche D, DiRaddo R, Del Maestro RF: NeuroTouch: a physics-based virtual simulator for cranial microneurosurgery training. Neurosurgery 71 (1 Suppl Operative):32-42, 2012

13. Gelberman RH, Szabo RM, Williamson RV, Hargens AR, Yaru NC, Minteer-Convery MA: Tissue pressure threshold for peripheral nerve viability. Clin Orthop Relat Res (178):285-291, 1983

14. Gélinas-Phaneuf N, Choudhury N, Al-Habib AR, Cabral A, Nadeau E, Mora V, et al: Assessing performance in brain tumor resection using a novel virtual reality simulator. Int $\mathbf{J}$ CARS 9:1-9, 2014

15. Gélinas-Phaneuf N, Del Maestro RF: Surgical expertise in neurosurgery: integrating theory into practice. Neurosurgery 73 (Suppl 1):30-38, 2013

16. Hanna GB, Elamass M, Cuschieri A: Ergonomics of hand-assisted laparoscopic surgery. Semin Laparosc Surg 8:92-95, 2001

17. Keir PJ, Bach JM, Rempel DM: Effects of finger posture on carpal tunnel pressure during wrist motion. J Hand Surg Am 23:1004-1009, 1998

18. Lee SH: Pianists' hand ergonomics and touch control. Med Probl Perform Art 5:72-78, 1990

19. Lemos JD, Hernandez AM, Soto-Romero G: An instrumented glove to assess manual dexterity in simulation-based neurosurgical education. Sensors (Basel) 17:988, 2017

20. Luchetti R, Schoenhuber R, Alfarano M, Deluca S, De Cicco G, Landi A: Carpal tunnel syndrome: correlations between pressure measurement and intraoperative electrophysiological nerve study. Muscle Nerve 13:1164-1168, 1990

21. Sawaya R, Bugdadi A, Azarnoush H, Winkler-Schwartz A, Alotaibi FE, Bajunaid K, et al: Virtual reality tumor resection: the force pyramid approach. Oper Neurosurg (Hagerstown) 14:686-696, 2017

22. Visser B, De Looze M, De Graaff M, Van Dieën J: Effects of precision demands and mental pressure on muscle activation and hand forces in computer mouse tasks. Ergonomics 47:202-217, 2004

23. Winkler-Schwartz A, Bajunaid K, Mullah MAS, Marwa I, Alotaibi FE, Fares J, et al: Bimanual psychomotor performance in neurosurgical resident applicants assessed using NeuroTouch, a virtual reality simulator. J Surg Educ 73:942-953, 2016

\section{Disclosures}

The authors report no conflict of interest concerning the materials or methods used in this study or the findings specified in this paper.

\section{Author Contributions}

Conception and design: Sawaya, Bajunaid, Sabbagh, Del Maestro. Acquisition of data: Bajunaid. Analysis and interpretation of data: Sawaya, Alsideiri, Bugdadi, Winkler-Schwartz, Azarnoush, Del Maestro. Drafting the article: Sawaya, Del Maestro. Critically revising the article: all authors. Reviewed submitted version of manuscript: all authors. Approved the final version of the manuscript on behalf of all authors: Sawaya. Statistical analysis: Sawaya. Administrative/technical/material support: Del Maestro. Study supervision: Del Maestro.

\section{Correspondence}

Robin Sawaya: Neurosurgical Simulation Research and Training Centre, Montreal Neurological Institute and Hospital, McGill University, Montreal, QC, Canada.robin.sawaya@mail.mcgill.ca. 